\title{
Bedeutung von Trägersystemen für die kutane Applikation von Sphingolipid-Präkursoren
}

\author{
Importance of Carrier Systems for Cutaneous Application of Sphingolipid \\ Precursors
}

\section{Zusammenfassung}

Die Substanzklasse der Sphingolipide, eine der Hauptkomponenten der Oberhaut, bietet neue Ansätze zur Behandlung trockener Haut. Durch Supplementierung von Sphingolipiden bzw. geeigneter Präkusoren soll die gestörte Hautbarriere bei trockener Haut rekonstruiert werden. Rationale für diesen Forschungsansatz sind die in den letzten Jahren gewonnenen klinischen Erkenntnisse zum atopischen Ekzem und - zumindest insbesondere auch - den Ichthyosen. Diese haben gezeigt, dass eine Störung der epidermalen Lipidzusammensetzung die Barrierestörung verursacht. Wesentlich für eine erfolgreiche Rekonstitution der gestörten Hautbarriere bei trockener Haut ist auch die Verwendung eines geeigneten Trägersystems, mit dem ein SphingolipidPräkursor in die Haut eingeschleust werden kann. Dies verdeutlichen die Ergebnisse aktueller Studien mit einer neuen Oleosomen-Technologie.
Abstract

Sphingolipids are one of the main components of the epidermis and offer new approaches in the care of dry skin. By supplementation of sphingolipids or adequate precursors, the defective barrier function of dry skin is to be reconstructed. Rationale for this research attempt are clinical insights obtained for atopic dermatitis and ichthyosis in the last years. They show that changes in stratum corneum lipids result in a defective barrier function. Essential for an effective reconstruction of the defective barriere function of dry skin is the use of practical carrier systems for carrying sphingolipid precursors into skin. Results of current studies with a new oleosome technology support this idea.

und qualitative Veränderung in der Lipidzusammensetzung bei atopischem Ekzem zu einem erhöhten Feuchtigkeitsverlust über die Haut. Es wird angenommen, dass durch die abnorme Expression einer so genannten Sphingomyelin-Acylase ein Mangel an bestimmten Strukturbestandteilen, den Ceramiden, Derivaten des Sphingosins, in der Epidermis entsteht [2]. Bei der Ichthyose konnte gezeigt werden, dass verschiedene Formen der Erkrankung einen verminderten Gehalt an Acylceramidfraktionen zeigen. Speziell bei der autosomal rezessiven Ichthyose liegen Veränderungen der Ceramidzusammensetzung vor. 
Ceramiden, hervorgerufen wird, führt offensichtlich zu einer Schwächung der Hautbarriere.

Die Arbeitsergebnisse verschiedener Forschergruppen lassen erkennen, dass auch bei trockener Haut der Ceramidgehalt vermindert ist. Gleichzeitig liegt eine andere lipidische Komponente der oberen Hautschicht, das Cholesterol, vermehrt vor.

Bei den Ceramiden handelt es sich um eine Gruppe unterschiedlicher Sphingolipide, die sich in der Haut vor allem in der obersten Epidermisschicht, dem Stratum corneum, befinden. Es wird heute von 8 verschiedenen Ceramidklassen (Ceramid 1-8) ausgegangen, die sich in ihrem Aufbau hinsichtlich der Sphingosinbasen und der mit der Kopfgruppe verknüpften Fettsäure unterscheiden (Abb. 1). Die Ceramide stellen mit bis zu 50 Prozent neben Cholesterol und freien Fettsäuren den größten Anteil der Stratum corneum Lipide dar [3]. Diese befinden sich in den interzellulären Zwischenräumen der Korneozyten. Die Korneozyten bestehen hauptsächlich aus unlöslichen gebündelten Proteinen und sind für die mechanische und chemische Struktur der Haut verantwortlich. Der interzellulären Lipidmatrix wird die eigentliche Barrierefunktion in Bezug auf das Eindringen fremder Substanzen sowie der Wasserhomöostase zugeschrieben. Die Struktur der Lipide, die ein hochgeordnetes multilamellares System bilden, ist für die Regulierung des Wasserhaushaltes von zentraler Bedeutung [4]. Dabei sind die vorhandenen Ceramide in der Lage, bei Anwesenheit von freien Fettsäuren und Cholesterol stabile Doppelschichten zu bilden. Von entscheidender Bedeutung für die Struktur der Doppelschichten ist das Ceramid 1, dessen $\omega$-Hydroxyfettsäure eine Doppelschicht komplett durchspannt. Der Linolsäurerest ragt dabei in die benachbarte Doppelschicht hinein, wodurch das System gefestigt wird. Eine Stabilisierung der Lipidmatrix wird ebenfalls durch einen hohen Cholesterolgehalt erzielt. Es zwingt benachbarte Fettsäureketten in eine regelmäßige Anordnung und erhöht damit die Ordnung des Systems [5]. Es wird angenommen, dass eine Störung der Ordnung zu einer Veränderung des Wassergleichgewichtes führt.

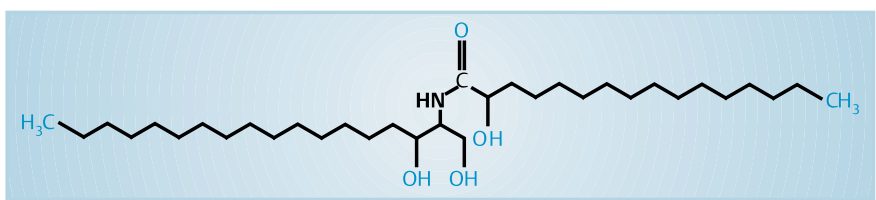

Abb. 1 Strukturformel von Sphinganin.

So zeigen etwa Untersuchungen des lamellaren Systems bei atopischer Dermatitis, dass im Vergleich zu gesundem Stratum corneum die strukturelle Anordnung der Lipide verändert ist. Dabei ist der Anteil an hexagonal angeordneten Lipidkristallen (Gelphase) im Vergleich zu einer orthorhombischen Packung (kristalline Phase) erhöht. Auch bei der Ichthyose ist der Anteil hexagonal angeordneter Lipide dominant [6].

Dieses Ergebnis zeigt, dass durch Erkrankung der Haut verschiedene Strukturzustände der Lamellenlipide auftreten können. Eine Störung des Gleichgewichtes zwischen der hexagonalen und der orthorombischen Anordnung, die durch eine veränderte Lipidzusammensetzung, insbesondere durch einen Mangel an
Applikation von Hautlipid-Gemischen verbessert Symptome trockener Haut

Grundvoraussetzung für die Rekonstruktion der gestörten Hautbarriere ist demnach die Wiederherstellung des Lipidgleichgewichtes. Die topische Supplementierung von Sphingolipiden bzw. geeigneter Präkursoren kann diesen Prozess möglicherweise fördern.

Zur Supplementierung von Sphingolipiden liegt eine Vielzahl von Studiendaten vor, die zu unterschiedlichen Ergebnissen kamen. So wurde in einer Studie die Wirksamkeit eines mit 0,2\% Ceramid IIIb angereicherten Hautpflegemittel auf die Barrierefunktion der Haut untersucht. Als Zielparameter wurde eine Verbesserung des transepidermalen Wasserverlustes (TEWL) definiert. Es zeigte sich jedoch, dass mit dieser Formulierung kein positiver Effekt auf den TEWL erreicht werden konnte [7].

Die Supplementierung einzelner Hautlipide scheint demnach nicht ausreichend zu sein, um eine Störung der Hautfunktion zu beheben. Dagegen trägt der Einsatz einer Formulierung, die eine Mischung aller relevanten Barrierelipide enthält, zu einer Senkung des transepidermalen Wasserverlustes bei atopischer Dermatitis bei. Dies konnte in einer Studie von Chamlin et al. belegt werden [8]. Eingeschlossen waren 24 Patienten mit atopischer Dermatitis, die 21 Wochen lang ihr Hautpflegemittel durch die Lipid-Mischung ersetzten. Im Abstand von drei Wochen wurde der Schweregrad der atopischen Dermatitis (SCORAD: Score für Schwere der atopischen Dermatitis) sowie verschiedene biophysikalische Funktionen des Stratum corneum bestimmt. Der SCORAD hatte sich nach 3 Wochen bei 22 der 24 Patienten signifikant verbessert. Die Verbesserung nahm zwischen der 6. und 20. bzw. 21. Woche bei allen Patienten zu. Der TEWL, der an betroffenen und nicht betroffenen Hautpartien bestimmt wurde, nahm parallel mit dem SCORAD ab. Sowohl die Kohäsion des Stratum corneum als auch die Hydratation verbesserten sich unter der Therapie langsam aber signifikant. Das mit der ceramidhaltigen Lipid-Mischung behandelte Gewebe wies extrazelluläre lamellare Membranen auf, die in den unbehandelten Hautpartien kaum vorkamen. Es zeigte sich auch, dass mit dem neuartigen Pflegemittel sogar der Gebrauch von Hautarzneimitteln eingespart werden konnte. Die Studie bestätigt, dass Mischungen von Barrierelipiden geeignet sind, die Hautbarriere zu verbessern. Weitere Studien sind jedoch notwendig, um eine optimale Mischung aus Hautlipiden zu finden.

\section{Präkursor Sphinganin beeinflusst Ceramidgehalt der Haut}

Einen Hinweis auf geeignete Präkursoren für trockene Haut bietet der Syntheseweg der Sphingolipide (Abb. 2). Er beginnt mit einer Kondensation von L-Serin und Palmitoyl-CoA am endoplasmatischen Retikulum, wodurch 3-Keto-Sphinganin gebildet wird. Dieses wird unter Einfluss einer NAD(P)H-abhängigen Reduktase zu Sphinganin reduziert. Im weiteren Verlauf entstehen Ceramide, die das zentrale Molekül der Sphingolipid-Biosynthe- 


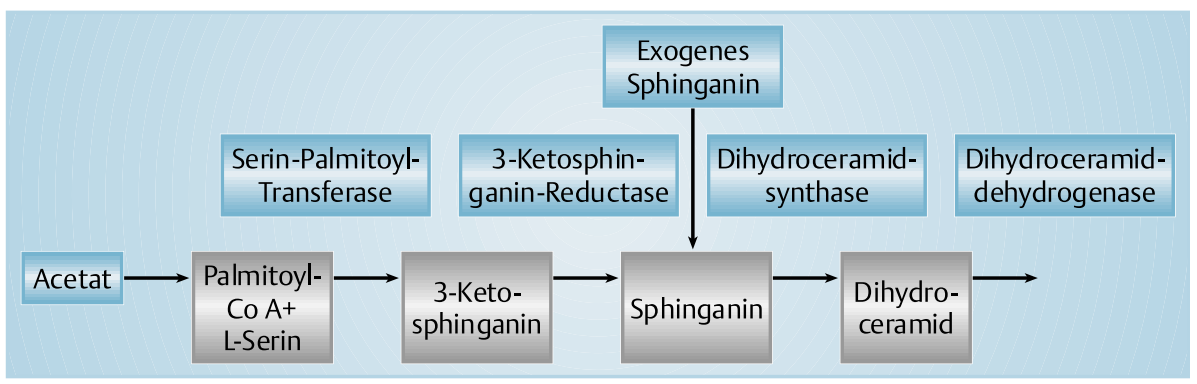

Abb. 2 Syntheseweg der Sphinganine und Ceramide in der Haut: Ansatzpunkt für Sphinganin-Zufuhr

se darstellen [9]. In der wissenschaftlichen Literatur werden als geeignete Präkursoren insbesondere Sphingosin, das Phytosphingosin, das Sphinganin und das 6-Hydroxy-Sphingosin diskutiert. Für Sphinganin (N-2-Hydroxyhexadecanoyl-Sphinganin) als Präkusor bei trockener Haut liegen bereits Studiendaten vor. So konnte in einer In-Vitro-Studie von L'Oréal-Recherche an einem Hautmodell (EPISKIN ${ }^{\mathrm{TM}}$ ) gezeigt werden, dass die Haut in der Lage ist, exogenes Sphinganin biosynthetisch in endogene Sphingolipide umzuwandeln. Im Rahmen der Studie wurde eine rekonstruierte Epidermis über 7 bzw. 14 Tage unter dem Einfluss von exogenem Sphinganin kultiviert. Die Quantifizierung der epidermalen Lipide mittels HPTLC (High Performance Thin Layer Chromatography) zeigte einen Anstieg des Gesamtgehaltes an epidermalen Ceramiden. Insbesondere die Gehalte der Ceramide 1, 2 und 3 stiegen unter dem Einfluss des exogenen Sphinganins an. Die anderen Lipidfraktionen blieben unverändert. Der Ablauf der Lipidsynthese wurde durch die Gabe exogenen Sphinganins nicht beeinflusst, wie Vergleichsuntersuchungen mit ${ }^{14} \mathrm{C}$-markiertem Acetat zeigen [10].

\section{Oleosome als geeignete Trägersysteme für Sphingolipid- Präkursoren}

Entscheidend für die effektive Aufnahme von Präkursoren in die Haut ist deren Transport zu den Hautschichten, in denen die Synthese von Ceramiden stattfindet. Untersuchungen haben gezeigt, dass ein Niosomen-Präparat verglichen mit Liposomen besser geeignet ist, einen zu untersuchenden Arzneistoff in die Haut zu schleusen. Vor diesem Hintergrund wurde die so genannte Oleosomen-Technologie etabliert. Im Gegensatz zu Liposomen stellen die ca. $250 \mathrm{~nm}$ großen Oleosomen Transportvehikel speziell für lipophile Wirkstoffe dar (Abb.3). Sie sind von einer konzentrisch angeordneten lamellaren Flüssigkristallphase aus amphiphilen Substanzen umgeben und transportieren in ihrem Inneren die Ölphase.

Im Rahmen klinischer Studien wurde die Wirkung einer sphinganinhaltigen Präparation zur Hautpflege, deren Galenik auf einer Oleosomen-Technologie (Nutrilogie ${ }^{\circledR}$ ) basiert, auf die trockene Haut untersucht.

An einer klinischen experimentellen Studie nahmen 30 Frauen im Alter zwischen 30 und 50 Jahren teil. Insgesamt 4 Wochen lang wurde das Präparat zweimal täglich auf das Gesicht und jeweils ein Bein appliziert. Das andere Bein blieb zur Kontrolle unbehandelt. Die Dermatologen bestimmten bei Studienbeginn sowie nach 4 und 5 Wochen die Hauttrockenheit anhand eines Score-Systems. Dafür wurde das Prüfareal in 10 Zonen kartiert,

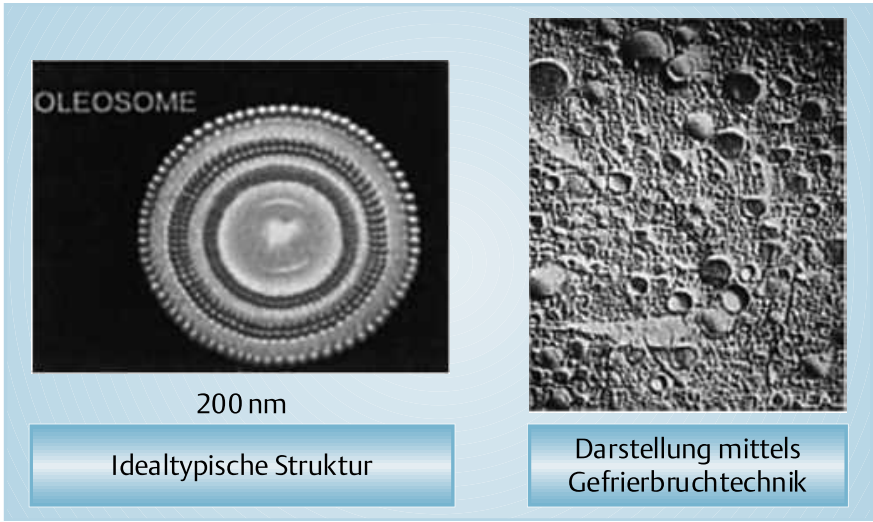

Abb. 3 Oleosome als Trägersysteme.

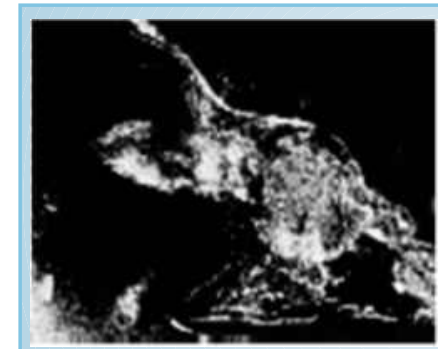

Tag 0

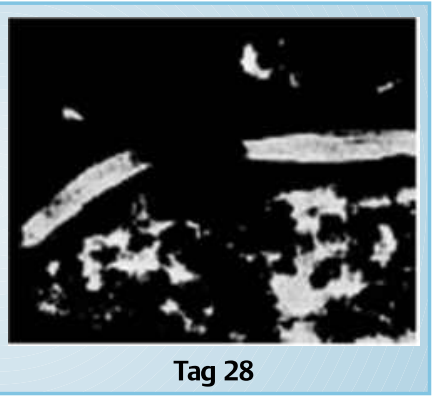

Tag 28
Abb. 4 Elektronenmikroskopische Aufnahme menschlicher Haut vor und 4 Wochen nach Beginn der Anwendung einer Oleosom-Sphingolipid-Zubereitung [11].

in denen die Werte zwischen Grad 0 (keine Abschuppung, normale Haut) und Grad 3 (schwere Abschuppung) unterschieden werden. Der Gesamtscore kann dabei einen Maximalwert von 30 erreichen.

Darüber hinaus wurde die Hautoberfläche mittels Videomikroskop sowie abgelöste Epidermis der Beine mit Hilfe eines Elektronenmikroskops bei Studienbeginn sowie nach 4 Wochen untersucht.

Nach 4 Wochen hatte sich der Score für die trockene Haut merklich verbessert (Gesicht um 43\%, Beine um 70\%, p <0,05). Die Verbesserung war auch noch eine Woche nach der Anwendung messbar. Zudem konnte nach vierwöchiger Anwendung im Elektronenmikroskop eine verbesserte Organisation der Lipidlamellen sichtbar gemacht werden (Abb. 4) [11]. 
Bestätigung fanden diese Studienergebnisse in einer klinischen Multicenterstudie mit 100 Frauen im Alter von 18 bis 60 Jahren. Die Probandinnen applizierten das Sphinganin-Oleosomen-Präparat vier Wochen lang zweimal täglich im Gesicht. Bewertet wurden die Hauttrockenheit, die Rauigkeit sowie die Flexibilität der Haut zu Studienbeginn sowie nach vier Wochen. Des Weiteren fand auch eine Analyse mittels Squamometrie Anwendung. Der Score für Hauttrockenheit verbesserte sich merklich um $65 \%(\mathrm{p}<0,05)$. Auch die Rauigkeit der Haut nahm im Vergleich zu Studienbeginn nach vierwöchiger Behandlung deutlich ab (Senkung um 77\%). Unter der Behandlung konnte die Flexibilität der Haut um 34\% gesteigert werden. Zudem wiesen alle Probandinnen nach vier Wochen normale Squamometriewerte auf. Hier lag nicht nur eine Reduktion in den Parametern für gestörte Haut vor, sondern es konnte als wichtiger Endpunkt eine Normalisierung der Haut ausgemacht werden [11]. Auch die Stiftung Warentest war von ihren Untersuchungsergebnissen überzeugt und bewertete die Creme ,Nutrilogie gegen trockene Haut' mit „sehr gut“. Es stehen demnach aktuell in Form der beiden Cremes mit Sphinganin, Nutrilogie ${ }^{\circledast}$ I und II, Präparate zur Verfügung, die zur Pflege der trockenen Haut in neuartiger Weise beizutragen vermögen.

\section{Literatur}

${ }^{1}$ Harding CR. The stratum corneum: structure and function in health and disease. Dermatol Ther 2004; 17: 1S6 - 1S15

${ }^{2}$ Murata Y, Ogata J, Higaki Y, Kawashima M, Yada Y, Higuchi K, Tsuchiya T, Kawainami S, Imokawa G. Abnormal expression of sphingomyelin acylase in atopic dermatitis: an etiologic factor for ceramide deficiency? J Invest Dermatol 1996; 106: 1242-1249

${ }^{3}$ Wertz PW. The nature of the epidermal barrier: biochemical aspects. Adv Drug Delivery 1996; 18: 283 - 294

${ }^{4}$ Wertz PW. Lipids and barrier function of the skin. Acta Derm Venerol 2000; 208: 7-11

${ }^{5}$ Wertz PW. Epidermal lipids. Semin Dermatol 1992; 11: 106-113

${ }^{6}$ Pilgram GS, Vissers DC, van der Meulen H, Pavel S, Lavrijsen SP, Bouwstra JA, Koerten HK. Aberrant lipid organization in stratum corneum of patients with atopic dermatitis and lamellar ichthyosis. J Invest Dermatol 2001; 117: 710 - 717

${ }^{7}$ De Paepe K, Derde MP, Roseeuw D, Rogiers V. Incorporation of ceramide $3 \mathrm{~b}$ in dermatocosmetic emulsions: effect on the transepidermal water loss of sodium lauryl sulphate-damaged skin. J Eur Acad Dermatol Venereol 2000; 14: $243-244$

${ }^{8}$ Chamlin SL, Kao J, Frieden IJ, Sheu MY, Fowler AJ, Fluhr JW, Williams ML, Elias PM. Ceramide-dominant barrier repair lipids alleviate childhood atopic dermatitis: changes in barrier function provide a sensitive indicator of disease activity. J Am Acad Dermatol 2002; 47: 198 - 208

${ }^{9}$ Merrill AH, Wang E. Enzymes of ceramide biosynthesis. Methods Enzymol 1992; 209: 427-437

10 Castiel-Higounenc I, Ferraris C, Lavalle A, Philippe M, Gaetani Q Schmidt R. Exogenous sphinganin derived sphingolipid increases epidermal ceramide content in human reconstructed skin, Poster EADV 1999

${ }^{11}$ Pierard GE, Korting HC, Montasier C, Marion C, Bouloc A. Dry skin improvement by an oleosome emulsion as a carrier for sphingolipid. Poster. $20^{\text {th }}$ World Congress of Dermatology, Paris, 2002 (P 0450). 